\title{
FORMATION OF QUASAR NUCLEI IN THE HEARTS OF ULTRALUMINOUS INFRARED GALAXIES
}

\author{
Yoshiaki Taniguchi, ${ }^{1}$ Satoru Ikeuchi, ${ }^{2}$ and Yasuhiro Shioya ${ }^{1}$ \\ Received 1998 October 26; accepted 1999 January 22; published 1999 February 15
}

\begin{abstract}
We investigate whether or not supermassive black holes (SMBHs) with mass $\gtrsim 10^{8} M_{\odot}$ can be made in the hearts of ultraluminous infrared galaxies (ULIGs) during the course of mergers between/among gas-rich galaxies. (1) If one progenitor galaxy had a seed SMBH with mass of $\sim 10^{7} M_{\odot}$, this seed SMBH can grow up to $\gtrsim 10^{8}$ $M_{\odot}$ due to efficient Bondi-type gas accretion during the course of merger, given a gas density in the circumnuclear region of $n_{\mathrm{H}} \sim 10^{3} \mathrm{~cm}^{-3}$. (2) Even if there was no progenitor galaxy with a seed SMBH, star clusters with compact remnants (neutron stars and/or black holes) produced in the circumnuclear starbursts can merge into the merger center within a dynamical timescale of $\sim 10^{9}$ yr to form an SMBH with $\gtrsim 10^{8} M_{\odot}$. Note, however, that the contribution of compact remnants supplied from hidden star clusters is necessary to lead to the formation of an SMBH. In conclusion, the ULIGs observed in the local universe can make SMBHs in their centers during the course of merging either by gas accretion onto a seed SMBH or by dynamical relaxation of compact remnants made in the violent circumnuclear starbursts. Therefore, it is quite likely that the ULIGs will finally evolve to optically luminous quasars, as suggested by Sanders et al.
\end{abstract}

Subject headings: galaxies: interactions — galaxies: starburst — galaxies: star clusters — infrared: galaxies — quasars: general

\section{INTRODUCTION}

Since the discovery of ultraluminous infrared galaxies (ULIGs; Soifer et al. 1984; Wright, Joseph, \& Meikle 1984; see Sanders \& Mirabel 1996 for a review), they have often been considered as possible precursors of optically bright quasars (Sanders et al. 1988a, 1988b; Norman \& Scoville 1988). This argument is based on the following observational properties of ULIGs. (1) Their bolometric luminosities amount to $\sim 10^{12} L_{\odot}$, being comparable to those of quasars (Sanders et al. 1988a). (2) Their luminosity function is similar to that of quasars in the local universe (Soifer et al. 1987; Sanders et al. 1988b). (3) All ULIGs are galaxy mergers or heavily interacting galaxies (Sanders et al. 1988a; Lawrence et al. 1989; Leech et al. 1994; Clements et al. 1996). Morphological evidence for galaxy mergers has also been obtained for a number of optically selected quasars in the local universe, although the majority are giant elliptical or giant elliptical-like galaxies (McLeod \& Rieke 1994a, 1994b; Disney et al. 1995; Bahcall et al. 1997; McLure et al. 1999). However, infrared-selected quasars tend to reside in morphologically disturbed hosts (e.g., Hutchings \& Neff 1988; Boyce et al. 1996; Baker \& Clements 1997). If most giant elliptical galaxies were formed by major mergers between/among disk galaxies (Toomre 1977; Barnes 1989; Ebisuzaki, Makino, \& Okumura 1991), it is possible that the majority of quasar hosts are major merger remnants. (4) ULIGs in later merger phases tend to have active galactic nuclei (AGNs) on the average (Sanders et al. 1988b; Majewski et al. 1993; Borne et al. 1997). Although all of the above properties suggest an evolutionary link from ULIGs to optically bright quasars, its plausibility is still in question.

It is generally considered that the quasars are powered by the central engine of AGNs, i.e., disk-gas accretion onto a supermassive black hole (SMBH), and masses of SMBHs in quasar nuclei are estimated to be $M_{.} \gtrsim 10^{8} M_{\odot}$ (e.g., Rees

\footnotetext{
Astronomical Institute, School of Science, Tohoku University, Aoba, Sendai 980-8578, Japan.

${ }^{2}$ Physics Department, School of Science, Nagoya University, Chikusa, Nagoya $464-8602$, Japan.
}

1984; Blandford 1990). Therefore, if an evolutionary link exists between ULIGs and quasars, we have to explain either the presence or the formation of SMBHs with mass higher than $\sim 10^{8} M_{\odot}$ in the hearts of ULIGs. This issue was already discussed by Norman \& Scoville (1988). They investigated the fate of a coeval, massive star cluster of $4 \times 10^{9} M_{\odot}$ within the central $10 \mathrm{pc}$ region (see also Weedman 1983) and found that an SMBH can be formed in the hearts of ULIGs. However, recent high-resolution optical and near-infrared images of a number of ULIGs using the Hubble Space Telescope have shown that the intense star-forming regions are scattered in circumnuclear regions up to about a few kpc from the nucleus (Shaya et al. 1994; Scoville et al. 1998; Surace et al. 1998). Therefore, it still seems uncertain whether or not SMBHs with $\gtrsim 10^{8} M_{\odot}$ can be made during the course of merger evolution in ULIGs. In this Letter, we investigate this issue, taking actual observational properties of ULIGs into account.

\section{FORMATION OF QUASAR NUCLEI IN THE HEARTS OF ULIGS}

Morphological features of ULIGs suggest that most ULIGs come from mergers between or among galaxies (Sanders et al. 1988a; Taniguchi \& Shioya 1998). Another important property of the ULIGs is that they are very gas rich: e.g., $M_{\mathrm{H}_{2}} \sim 10^{10}$ $M_{\odot}$ (Sanders et al. 1988a; Scoville et al. 1991; Downes \& Solomon 1998). Therefore, the progenitor galaxies of ULIGs, such as giant spiral galaxies, are rich in gas. Since intense starbursts are observed in many ULIGs, the most probable formation mechanism of SMBHs is the collapse of compact remnants of massive stars (Weedman 1983; Norman \& Scoville 1988). Another important issue is whether or not progenitor galaxies had SMBHs originally in their nuclei. Although masses of SMBHs in nearly spiral galaxies (i.e., progenitor galaxies of ULIGs) are of the order of $10^{6}-10^{7} M_{\odot}$ at most (e.g., Kormendy et al. 1998 and references therein), these seed SMBHs could grow due to gas accretion in circumnuclear dense-gas regions during the course of the merger. Therefore, we consider two cases: (1) at least one progenitor had an SMBH with $M_{\bullet} \sim 10^{6}-10^{7} M_{\odot}$, and (2) no progenitor had an SMBH. 


\subsection{Mergers between/among Nucleated Galaxies}

If a progenitor galaxy had an SMBH in its nucleus, this seed SMBH could grow in mass during the course of a merger because the central region of a ULIG is very gas rich. Such gas accretion may be efficient within the central $1 \mathrm{kpc}$ region because the gas density is quite high in the central region, i.e., $\sim 10^{3}-10^{4} \mathrm{~cm}^{-3}$ (Scoville, Yun, \& Bryant 1997; Downes \& Solomon 1998). We here consider the classical Bondi-type (Bondi 1952) gas accretion onto the SMBH. This gas accretion rate is given by

$$
\dot{M}=2 \pi m_{\mathrm{H}} n_{\mathrm{H}} r_{a}^{2} v_{e}
$$

where $m_{\mathrm{H}}, n_{\mathrm{H}}, r_{a}$, and $v_{e}$ are the mass of a hydrogen atom, the number density of the hydrogen atom, the accretion radius defined as $r_{a}=G M . v_{e}^{-2}(M$. is the mass of the seed SMBH), and the effective relative velocity between the seed SMBH and the ambient gas, respectively. A typical dynamical mass of the central $1 \mathrm{kpc}$ region is of the order of $\sim 10^{9} M_{\odot}$. Suppose that the SMBH with mass of $10^{6} M_{\odot}$ is sinking toward the dynamical center of the merger. Its orbital velocity is $v_{\text {orb }} \simeq 67 M_{\text {nuc, } 9}^{1 / 2} r_{1}^{-1 / 2} \mathrm{~km} \mathrm{~s}^{-1}$, where $M_{\text {nuc, } 9}$ is the dynamical mass of the central $1 \mathrm{kpc}$ region of the merger and $r_{1}$ is the radius in units of $1 \mathrm{kpc}$. The crossing time of the SMBH is $T_{\text {cross }} \sim$ $10^{7} \mathrm{yr}$. Therefore, the merging timescale is estimated to be $T_{\text {merger }} \sim 10 T_{\text {cross }} \sim 10^{8} \mathrm{yr}$ (e.g., Barnes 1989). Adopting an average gas density in the circumnuclear regions of ULIGs to be $n_{\mathrm{H}} \sim 10^{3} \mathrm{~cm}^{-3}$, we obtain the accreting gas mass during the course of a merger:

$$
M_{\mathrm{acc}}=\dot{M} T_{\text {merger }} \sim 3 \times 10^{5} M_{\bullet, 6}^{2} n_{\mathrm{H}, 3} v_{e, 100}^{-3} T_{\text {merger }, 8} M_{\odot},
$$

where $M_{,, 6}$ is the mass of SMBH in units of $10^{6} M_{\odot}, n_{\mathrm{H}, 3}$ is the average gas density in units of $10^{3} \mathrm{~cm}^{-3}, v_{e, 100}$ is the orbital velocity with respect to the ambient gas in units of $100 \mathrm{~km}$ $\mathrm{s}^{-1}$, and $T_{\text {merger, } 8}$ is the merger timescale in units of $10^{8} \mathrm{yr}$. This estimate implies that the seed SMBH cannot grow up to $M_{\odot} \gtrsim 10^{8} M_{\odot}$ if the seed SMBH is less massive than $10^{7} M_{\odot}$. Since the ULIGs come from mergers between two galaxies or among several galaxies, their progenitor galaxies should be very giant spiral galaxies in order to pile up molecular gas up to $\sim 10^{10} M_{\odot}$ in their central regions (e.g., Sanders et al. 1988a). In fact, nearby spiral galaxies such as M31 and NGC 4258 have SMBHs with a few $10^{7} M_{\odot}$ (e.g., Kormendy et al. 1998 and references therein; see also Miyoshi et al. 1995 for the case of NGC 4258). Therefore, it seems quite likely that the seed SMBH may be more massive than that adopted in the above estimate. If the seed SMBH is more massive than a few times $10^{7} M_{\odot}$, it could grow up to $\gtrsim 10^{8} M_{\odot}$. Although we have no knowledge about the seed SMBHs in the progenitors, our estimates given here suggest that the gas accretion in the dense gas clouds onto the seed SMBH can lead to the formation of a quasar nucleus in the hearts of ULIGs.

\subsection{Mergers between/among Nonnucleated Galaxies}

Next we consider a case in which there is no seed SMBH in the progenitor galaxies of ULIGs. In this case, a possible way to form quasar nuclei in ULIGs is to pile up the circumnuclear star clusters of compact remnants of massive stars: black holes and neutron stars, each of which has a few $M_{\odot}$ at most. The pioneering discussion on this issue was given by
Weedman (1983). Using both starburst models by Gehrz, Sramek, \& Weedman (1983) and optical spectroscopic observations (i.e., $\mathrm{H} \alpha$ luminosity), he suggested that starburst galaxies with $L(\mathrm{H} \alpha) \gtrsim 10^{42}$ ergs $\mathrm{s}^{-1}$ could produce compact starburst remnants up to $\sim 10^{9} M_{\odot}$. However, since the $\mathrm{H} \alpha$ luminosity of starburst galaxies is dominated by the most massive stars in the starburst region, it seems hard to estimate the total mass of compact remnants solely using $L(\mathrm{H} \alpha)$. Thus, the estimate by Weedman (1983) may provide a rough upper limit for the compact remnant mass in the starburst galaxies. Norman \& Scoville (1988) also discussed the formation of quasar nuclei in ULIGs. However, their assumption (a coeval, massive star cluster of $4 \times 10^{9} M_{\odot}$ within the central $10 \mathrm{pc}$ region) turns out to be unlikely because the recent high-resolution optical and near-infrared imaging by the Hubble Space Telescope of ULIGs have shown that blue star clusters are distributed in the circumnuclear regions up to $r$ approximately a few kpc (Shaya et al. 1994; Surace et al. 1998; Scoville et al. 1998). Although the central star cluster associated with the western nucleus of Arp 220 is very luminous and its mass is estimated to be $\gtrsim 10^{8}$ $M_{\odot}$, typical masses of the circumnuclear star clusters are of the order of $M_{\mathrm{cl}} \sim 10^{7} M_{\odot}$ at most (Shaya et al. 1994; Scoville et al. 1998; Shioya, Taniguchi, \& Trentham 1999; see also Taniguchi, Trentham, \& Shioya 1998). Although some star clusters may be hidden because of heavy extinction (Scoville et al. 1991; Genzel et al. 1998), we first investigate whether or not the star clusters found both in the optical and in the near-infrared (NIR; Shaya et al. 1994; Scoville et al. 1998) will be responsible for the formation of an SMBH with $M$. $\gtrsim 10^{8}$ $M_{\odot}$.

Shaya et al. (1994) discussed the fate of circumnuclear star clusters; since these clusters will lose their kinetic energy to individual stars during random encounters (i.e., dynamical friction), they will sink toward the merger center within $\sim 10^{9} \mathrm{yr}$. Here, from a viewpoint of dynamical relaxation of the star clusters, we examine whether or not compact remnants formed in the circumnuclear star-forming clusters can make an SMBH with $M_{.} \sim 10^{8} M_{\odot}$. For simplicity, we consider a case in which 10 circumnuclear star-forming clusters, each of which has a total stellar mass of $10^{7} M_{\odot}$, are distributed within $r$ approximately a few kpc. It is known that stars with $m_{*} \geq 8 M_{\odot}$ produce compact remnants. We estimate how many such massive stars are formed in each cluster.

We assume that stars are formed with a Salpeter-like initial mass function,

$$
\phi(m)=\beta m^{-\mu}
$$

where $m$ is the stellar mass in units of $M_{\odot}$ and $\beta$ is a normalization constant determined by the relation

$$
\int_{m_{l}}^{m_{u}} \phi(m) d m=1,
$$

which leads to

$$
\beta=\frac{(\mu-1) m_{l}^{\mu-1}}{1-\left(m_{l} / m_{u}\right)^{\mu-1}}
$$

The number of stars with a mass range $m_{1} \leq m_{*} \leq m_{2}$ is esti- 
mated as

$$
N\left(m_{1} \leq m_{*} \leq m_{2}\right)=\int_{m_{1}}^{m_{2}} \frac{\phi(m)}{m} d m
$$

Using $\beta$ in equation (5), we rewrite equation (6) as

$$
N\left(m_{1} \leq m_{*} \leq m_{2}\right)=\left(\frac{\beta}{\mu}\right)\left(m_{1}^{-\mu}-m_{2}^{-\mu}\right) \text { stars } M_{\odot}^{-1} .
$$

There are three free parameters; the power index $(\mu)$ and the upper and lower mass limits of the initial mass function $\left(m_{u}\right.$ and $\left.m_{l}\right)$. Since stars with $m_{*} \geq 8 M_{\odot}$ produce compact remnants, we set $m_{1}=8 M_{\odot}$ and $m_{2}=m_{u}>m_{1}$. In Table 1 , we give results for some possible combinations of the parameters. Although $\mu=1.35$ is the canonical value for stars in the solar neighborhood, there are some lines of evidence that massive stars are more preferentially formed in such violent star-forming regions, i.e., a top-heavy initial mass function with $m_{l} \sim 1 M_{\odot}$ (e.g., Goldader et al. 1997 and references therein). Therefore, we adopt a case of $\mu=0.35, m_{u}=30 M_{\odot}$, and $m_{l}=1 M_{\odot}$. In this case, there are $\sim 4 \times 10^{5}$ massive stars with $m_{*} \geq 8 M_{\odot}$ in each cluster. Each compact remnant has a mass from a few $M_{\odot}$ (for neutron stars) to several $M_{\odot}$ (for black holes). Therefore, the total mass of compact remnants in each cluster is $\sim 10^{6}$ $M_{\odot}$. These 10 clusters will be relaxed dynamically with a timescale of

$$
T_{\mathrm{dyn}} \sim n_{\mathrm{cl}}^{1 / 2} G^{-1 / 2} r_{\mathrm{cl}}^{3 / 2} M_{\mathrm{cl}}^{-1 / 2} \sim 9.4 \times 10^{8} r_{\mathrm{cl}, 1}^{3 / 2} M_{\mathrm{cl}, 7}^{-1 / 2}(\mathrm{yr}),
$$

where $r_{\mathrm{cl}, 1}$ is the typical size of a circumnuclear region with 10 star clusters in units of $1 \mathrm{kpc}$ and $M_{\mathrm{cl}, 7}$ is the mass of the clusters in units of $10^{7} M_{\odot}$. Therefore, we expect that an SMBH with mass of $\sim 10^{7} M_{\odot}$ will be made $\sim 10^{9}$ yr after the onset of circumnuclear starbursts in ULIGs. This mass is smaller than that necessary for quasar nuclei (i.e., $\gtrsim 10^{8} M_{\odot}$ ). Each cluster of compact remnants would be able to gain its mass by gas accretion, as discussed in $\S 2.1$. However, the accreted mass is estimated to be $\sim 3 \times 10^{7} M_{\odot}$ for 10 clusters in total, being still less massive than $10^{8} M_{\odot}$.

Here it should be again remembered that all star clusters in the central region of ULIGs cannot be observed in both the optical and the NIR because inferred extinction toward the nuclei of ULIGs is very large, e.g., $\gtrsim 50 \mathrm{mag}$ (Genzel et al. 1998; Scoville et al. 1991). Therefore, it is quite likely that the majority of nuclear star clusters in ULIGs are hidden by a large amount of gas and dust. Recently, Shioya et al. (1999) analyzed the optical-NIR spectral energy distributions of nuclear star clusters in Arp 220. They found that these clusters are more massive systematically (i.e., $\gtrsim 10^{8} M_{\odot}$ ) than circumnuclear ones but can account only for about one-seventh of the total bolometric luminosity of Arp 220. Although $\mathrm{OH}$ megamaser sources found in the central region of Arp 220 may provide possible evidence for hidden AGNs (Diamond et al. 1989; Lonsdale et al. 1998), the recent mid-infrared spectroscopy of a sample of ULIGs has shown that the majority of ULIGs such as Arp 220 are powered by nuclear starbursts (Genzel et al. 1998; Lutz et al. 1998). Therefore, it is strongly suggested that some hidden star clusters should be responsible for the remaining ( 6/7) bolometric luminosity of Arp 220. Since compact remnants produced in the hidden clusters will join to form
TABLE 1

The Number of Massive Stars Per Unit Mass $\left(M_{\odot}\right)$

\begin{tabular}{ccrcc}
\hline \hline \multicolumn{1}{c}{} & $\begin{array}{c}m_{l} \\
\left(M_{\odot}\right)\end{array}$ & $\begin{array}{c}m_{u} \\
\left(M_{\odot}\right)\end{array}$ & $\beta$ & $\begin{array}{c}N\left(m_{*} \geq 8 M_{\odot}\right) \\
\left(\operatorname{stars} M_{\odot}^{-1}\right)\end{array}$ \\
\hline $0.35 \ldots \ldots$ & 1 & 100 & 0.034 & 0.0278 \\
$0.35 \ldots \ldots$ & 1 & 30 & 0.080 & 0.0409 \\
$1.35 \ldots \ldots$ & 1 & 100 & 0.437 & 0.0189 \\
$1.35 \ldots \ldots$ & 1 & 30 & 0.503 & 0.0187 \\
\hline
\end{tabular}

an SMBH, it is expected that an SMBH with $M_{.} \gtrsim 10^{8} M_{\odot}$ will be formed in the hearts of ULIGs.

\section{DISCUSSION}

We have shown that an $\mathrm{SMBH}$ with mass $\gtrsim 10^{8} M_{\odot}$ can be made in a ULIG during the course of merger between/among gas-rich galaxies regardless of the presence of a seed SMBH in progenitor galaxies, i.e., (1) if one progenitor galaxy had a seed $\mathrm{SMBH}$ with mass of $\sim 10^{7} M_{\odot}$, this seed $\mathrm{SMBH}$ can grow up to $\gtrsim 10^{8} M_{\odot}$ because of efficient Bondi-type gas accretion during the course of merger given a gas density in the circumnuclear region of $n_{\mathrm{H}} \sim 10^{3} \mathrm{~cm}^{-3}$, and (2) even if there was no progenitor galaxy with a seed SMBH, the star clusters of compact remnants made in the circumnuclear starbursts can merge into the merger center within a dynamical timescale of $\sim 10^{9}$ yr to form an SMBH with $\gtrsim 10^{8} M_{\odot}$. Note, however, that the contribution of compact remnants supplied from hidden star clusters is necessary to explain the formation of SMBHs. In conclusion, the ultraluminous infrared galaxies observed in the local universe can make SMBHs in their centers during the course of merger either by the gas accretion onto a seed SMBH or by the dynamical relaxation of star clusters of compact remnants made in the violent circumnuclear starbursts.

The presence of an SMBH with $M_{\odot} \gtrsim 10^{8} M_{\odot}$ is a crucially important necessary condition for the occurrence of quasar activity. In the local universe, the masses of SMBHs in giant spiral galaxies (e.g., our Galaxy, M31, NGC 1068, and NGC 4258) are as low as $M_{\odot} \sim 10^{6}-10^{7} M_{\odot}$ (Kormendy et al. 1998, and references therein). Therefore, it is suggested that isolated, typical spiral galaxies cannot harbor quasar nuclei. However, mergers between/among gas-rich galaxies can cause efficient gas fueling toward the nuclear regions of the merging systems and then trigger intense starbursts either as a result of the piling of a lot of gas (Mihos \& Hernquist 1994) or by the dynamical effect of SMBH binaries (Taniguchi \& Wada 1996; Taniguchi \& Shioya 1998). Furthermore, as demonstrated in the present work, these mergers provide a possible way to form SMBHs with $M_{.} \gtrsim 10^{8} M_{\odot}$. In this respect, it is quite likely that the ULIGs will finally evolve to optically luminous quasars as suggested by Sanders et al. (1988a, 1988b).

Finally, it is worthwhile noting that some elliptical galaxies could be formed by galaxy mergers (e.g., Toomre 1977; Barnes 1989; Ebisuzaki et al. 1991). We also note that some elliptical galaxies (e.g., M87, NGC 3115, NGC 3377, NGC 4261, and so on) have SMBHs with $M$. $\gtrsim 10^{8} M_{\odot}$ (Kormendy et al. 1998, and references therein). Actually, investigating the physical conditions of ULIGs (i.e., mass density and velocity dispersion), Kormendy \& Sanders (1992) found evidence that ULIGs are elliptical galaxies forming by merger-induced dissipative collapse (see also Wright et al. 1990; Baker \& Clements 1997). Therefore, we suggest that almost all SMBHs with $M_{\mathbf{0}} \gtrsim 10^{8}$ $M_{\odot}$ in the local universe were made by galaxy mergers. 
We would like to thank an anonymous referee for his/her useful comments. This work was supported in part by the Min- istry of Education, Science, Sports, and Culture in Japan under grants 07055044,10044052 , and 10304013.

\section{REFERENCES}

Bahcall, J. N., Kirhakos, S., Saxe, D. H., \& Schneider, D. P. 1997, ApJ, 479, 642

Baker, A. C., \& Clements, D. L. 1997, in Extragalactic Astronomy in the Infrared, ed. G. A. Mamon, X. T. Trinh, \& J. T. V. Tran (Paris: Editions Frontières)

Barnes, J. E. 1989, Nature, 338, 123

Blandford, R. 1990, Active Galactic Nuclei, ed. R. D. Blandford, H. Netzer, \& L. Woltier (Berlin: Springer), 161

Bondi, H. 1952, MNRAS, 112, 195

Borne, K., et al. 1997, in Extragalactic Astronomy in the Infrared, ed. G. A. Mamon, X. T. Trinh, \& J. T. V. Tran (Paris: Editions Frontières)

Boyce, P. J., et al. 1996, ApJ, 473, 760

Clements, D. L., Sutherland, W. J., McMahon, R. G., \& Saunders, W. 1996, MNRAS, 279, 477

Diamond, P. J., Norris, R. P., Baan, W. A., \& Booth, R. S. 1989, ApJ, 340, L49

Disney, M. J., et al. 1995, Nature, 376, 150

Downes, D., \& Solomon, P. M. 1998, ApJ, 507, 615

Ebisuzaki, T., Makino, J., \& Okumura, S. K. 1991, Nature, 354, 212

Gehrz, R. D., Sramek, R. A., \& Weedman, D. W. 1983, ApJ, 267, 551

Genzel, R., et al. 1998, ApJ, 498, 579

Goldader, J. D., Joseph, R. D., Doyon, R., \& Sanders, D. B. 1997, ApJ, 474, 104

Hutchings, J. B., \& Neff, S. G. 1988, AJ, 96, 1575

Kormendy, J., Bender, R., Evans, A. S., \& Richstone, D. 1998, AJ, 115, 1823

Kormendy, J., \& Sanders, D. B. 1992, ApJ, 390, L53

Lawrence, A., Rowan-Robinson, M., Leech, K., Jones, D. H. P., \& Wall, J. V. 1989, MNRAS, 240, 329

Leech, K. J., Rowan-Robinson, M., Lawrence, A., \& Hughes, J. D. 1994, MNRAS, 267, 253

Lonsdale, C. J., Lonsdale, C. J., Diamond, P., \& Smith, H. E. 1998, ApJ, 493 , L13 (erratum 494, L239)

Lutz, D., Spoon, H. W. W., Rigopoulou, D., Moorwood, A. F. M., \& Genzel, R. 1998, ApJ, 505, L103

Majewski, S. R., Hereld, M., Koo, D. C., Illingworth, G. D., \& Heckman, T. M. 1993, ApJ, 402, 125
McLeod, K. K., \& Rieke, G. H. 1994a, ApJ, 420, 58 1994b, ApJ, 431, 137

McLure, R. J., Dunlop, J. S., Kukula, M. J., Baum, S. A., O’Dea, C. P., \& Hughes, D. H. 1999, ApJ, submitted (astro-ph/9809030)

Mihos, C. J., \& Hernquist, L. 1994, ApJ, 431, L9

Miyoshi, M., Moran, J., Herrnstein, J., Greenhill, L., Nakai, N., Diamond, P., \& Inoue, M. 1995, Nature, 373, 127

Norman, C., \& Scoville, N. 1988, ApJ, 332, 124

Rees, M. J. 1984, ARA\&A, 22, 471

Sanders, D. B., \& Mirabel, I. F. 1996, ARA\&A, 34, 749

Sanders, D. B., et al. 1988a, ApJ, 325, 74

Sanders, D. B., Soifer, B. T., Elias, J. H., Neugebauer, G., \& Matthews, K. 1988b, ApJ, 328, L35

Scoville, N. Z., et al. 1998, ApJ, 492, L107

Scoville, N. Z., Sargent, A. I., Sanders, D. B., \& Soifer, B. T. 1991, ApJ, 366, L5

Scoville, N. Z., Yun, M. S., \& Bryant, P. M. 1997, ApJ, 484, 702

Shaya, E., Dowling, D. M., Currie, D. G., Faber, S. M., \& Groth, E. J. 1994, AJ, 107, 1675

Shioya, Y., Taniguchi, Y., \& Trentham, N. 1999, ApJ, submitted

Soifer, B. T., et al. 1984, ApJ, 283, L1 1987, ApJ, 320, 238

Surace, J. A., Sanders, D. B., Vacca, W. D., Veilleux, S., \& Mazzarella, J. M. 1998, ApJ, 492, 116

Taniguchi, Y., \& Shioya, Y. 1998, ApJ, 501, L167

Taniguchi, Y., Trentham, N., \& Shioya, Y. 1998, ApJ, 504, L79

Taniguchi, Y., \& Wada, K. 1996, ApJ, 469, 581

Toomre, A. 1977, in The Evolution of Galaxies and Stellar Populations, ed. B. M. Tinsley \& R. B. Larson (New Haven: Yale University Observatory), 401

Weedman, D. W. 1983, ApJ, 266, 479

Wright, G. S., James, P. A., Joseph, R. D., \& McLean, I. S. 1990, Nature, 344, 417

Wright, G. S., Joseph, R. D., \& Meikle, W. P. S. 1984, Nature, 309, 31 